\title{
Energy Efficiency Analysis by Game Theoretic Approach in the Next Generation Network
}

\author{
Joydev Ghosh \\ School of Computer Science and Robotics, National Research Tomsk Polytechnic University, RUSSIA, Email: \\ joydev.ghosh.ece@gmail.com
}

\begin{abstract}
In downlink orthogonal frequency division multiple access (OFDMA) networks, an effective way of using the limited wireless spectrum resources can significantly improve network response. This paper presents a game-theoretic scheme with anticoordinated players by incorporating adaptation of femto base station (FBS) transmit power, attenuation of interference and utility function for open access mode and closed access mode respectively. The deployment of femtocells in the networks is to produce improved energy efficiency (EE) and optimized reponse of payoff function. In open access mode, each user belongs to the operator's network can connect to the FBS and in closed access case, only a specified set of users can privately couple to the FBS whereas in the early access scenario it only allows authentic subscribers to take the advantage of femtocell networks. Additionally, the operating principle of spectrum sharing scheme has been discussed in which FBS as a player acquire knowledge from utility responses of their strategic communications and revise their strategies at each level of the game process. Here, an FBS is regarded as a player in the game to select the users who are satisfied to a greatest extent and an FBS plays a role of mentor. Thereafter, the equilibrium concept has been invoked to aid the anti-coordinated players for the strategies. Besides, a femtocell power adaptation algorithm has also been introduced based upon the set of enabled femtocells who can be used to retain its blocking probability that guarantees convergence to the stable strategy of the game, where the FBS monitors the subscribers' actions and gives only limited data exchange. The simulations demonstrate that the proposed algorithm attains a high quality performance such as rapid convergence, interference attenuation to a greatest extent, noticeable $\mathrm{EE}$ improvement etc. Finally, validate the simulation results with its rarely studied extension in cognitive femtocell networks.
\end{abstract}

Index Terms-Energy Efficiency, Utility Function, Payoff Function, Access Mode, Non-cooperative Game Theory, Femtocell, Wireless Network.

\section{INTRODUCTION}

Drastically growing energy costs and risingly rigid scenario standards have brought to an emerging trend of addressing energy efficiency (EE) aspect of next generation networks [1]. In early days, more than two third of total energy was consumed by radio access technology in the wireless networks [2]. Thus, improvement of $\mathrm{EE}$ in the wireless networks is clearly an important concern to meet the large demand of mobile traffic. The cognitive radio (CR) technology takes a major role in increasing EE in wireless cellular networks [3]. The cognitive capability consists of a large range of features such as spectrum sensing (SS) [4], spectrum allocation [5] and adaptive power transmission [6] which are beneficial to acquire a balance among EE, Spectral Efficiency (SE), Bandwidth, and deployment efficiency in wireless radio access networks. Moreover, both economic and environmental aspects have been inspired an inclination to reduce the amount of energy draining. The cooperation between BS and subscriber is one of the promising way to save energy [7], [8]. The CR [9] is a technology which activates devices to find out which fragment of the spectrum are not properly used up, and to share them despite the fact that they are unlicensed. As of today, a lot of work has been demonstrated for the resource allocations and interference reduction techniques in future generation networks with small cell, where nearly all of them articulate on the orthogonal frequency division multiple access (OFDMA) resource allocations [10] and sub-channel allocations [11]. The CR technology [12], [23] activates femto user equipments (FUEs), where an user can connect to the FBS with low communication range rather than higher communication range to macro base station (MBS), to tune transmission variations depending upon the environmental conditions [13] and may be employed to resolve the power adaptation issues in cognitive femtocell networks [14], [24]. A FBS is a low power, small cellular base station, which can operate in both licensed and unlicensed band and it provides network service upto 100 meters distance [21], [25]. On the other side, the EE is becoming an influential factor in quantifying the evaluation of cognitive femtocell networks due to drastic rising energy prices and rigid scenario standards. Implantation of FBS is a promising solution as an energy-efficient technique for its capability to decrease the energy dissipation and to elongate battery life of handset due to the small gap between transmitter and receiver, and low transmit power property. In this regard, CR technology acts a crucial role to excel energy efficiency in HetNets [15], [26]. Hence, it is a consequential to review the cooperation between FBS and CR on EE in cognitive femtocell networks. Based on the cooperation between players, a game can be categorized into two types such as non-cooperative and cooperative games [16], [18]. In a non-cooperative game, individual player succeeds as a different rational entity to get the best use of its payoff and apply decisions independently [17]. Although, in a cooperative games, all players in a team act as a single entity, where players are not in any competition among one another rather they aim at optimizing the total payoff of the team. The satisfaction level of the players is usually defined by a utility function. The admission control that supervises the access policy is considered as an analytical issue in this network model. The open access mode is one of the admission control policies which allows several users to 
establish a communication link with the FBS networks. The closed access mode only allows the access permissions to the closed subscriber group (CSG) to establish a connection with the respective FBS networks. For the mobile users that are closed to adjacent FBSs but not connected may generate a large amount of interference to the femtocell for accessing the macrocell.

An analytical model development of a basic structure underlying a two-layer network is performed to investigate and probe the different metrics such as utility function, payoff function, access policy and energy efficiency in comparison to that of two-layers network where FBSs are grouped into different clusters at the macrocell edges as presented in [22]. Here this system model has been shown in contrast to the reference [22] that the networks where open and close access modes between a FBS and a user respectively have a substantial role in spectrum sharing cost and femtocell power adaptation even for wide-reaching macrocells. Extending from [22], utility function and payoff function obtained in a cellular network consisting FBSs as a cluster at the macrocell edges have been analytically demonstrated further by considering the significant roles of the access modes in the proposed network model. Besides, the trade-off studies of EE and payoff functions in the proposed network model have been performed to find stability point in the game by means of their concavity downward response, whereas the solution of stability condition in the game has been carried out by applying nash equilibrium is presented in [22]. In addition to the above, femtocell power adaptation algorithm is proposed articulating more on power fine-tuning at the BSs, whereas search algorithm has been introduced as in [22] for seeking the best strategy to deal with femto user's best response. In Particular, this paper presents the following three-fold contributions:

1) In order to weaken influential effect of interference and optimize the energy-efficient transmissions of MBSs, we utilize the cost of interference in dual-layer wireless networks to model the unwanted interference between femtocell and large cell that are extensively used to quantify the interference effect. The analytical framework takes into consideration for the number of macro user equipments (MUEs), FUE receiver's transmit power, and interference threshold. For energy-efficient cognitivefemtocell resource allocation, we introduce a femtocell power adaptation algorithm (FPAA) by applying game theory.

2) The utility function explicated based on the channel capacity of the FBS network to achieve optimum condition for OFDMA based cognitive femtocell networks. Numerical results encapsulate that the channel capacity of the subscribers under particular intervals of closed rate $\epsilon$ is larger than that of the closed access policy and open access policy. We develop both an analytical framework and an admission control prototype for joint resource allocation and dynamic spectrum sharing, respectively, in non-cooperative game theoretic based cognitive femtocell networks.

3) We articulate on the EE view of the frequency band

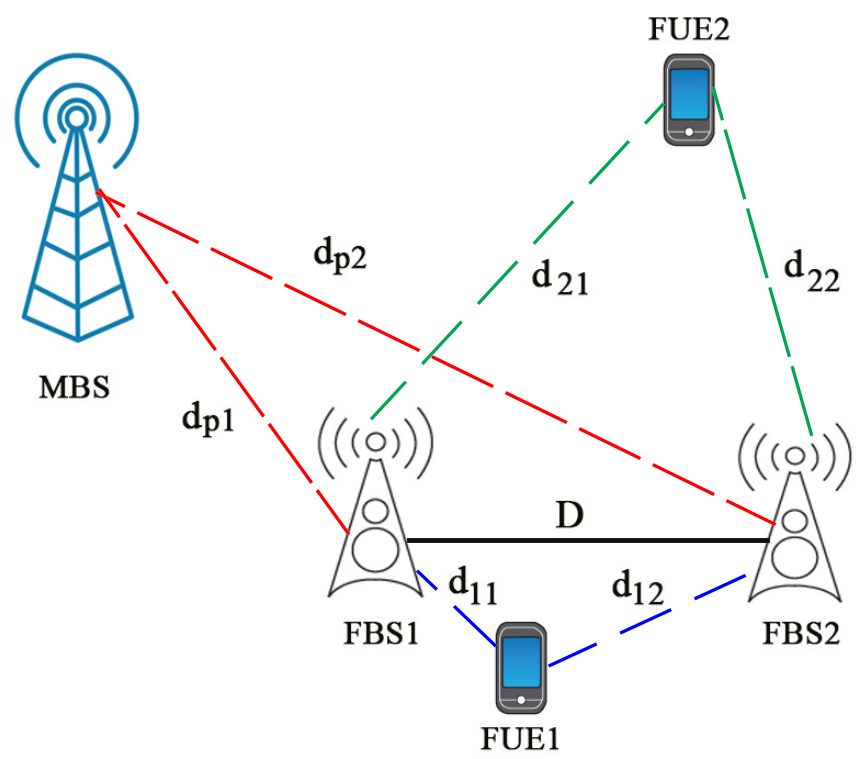

Fig. 1: A HetNet scenario where two FBS permits wireless access authority to FUE1 and FUE2

allotment and power assignment in cognitive femtocell networks. As both CR and FBS are potential technologies to increase $\mathrm{EE}$ in the next generation networks, the interplay among them excellences further research.

\section{SySTEM MOdEL}

In this section, a non-cooperative game theoretic method is introduced to adaptively allocate power in a two femtocell and one macrocell based HetNets. The aim is to optimize the network coverage with the limitation of the required signal to interference plus noise ratio (SINR) and the safety of an MUE ${ }^{1}$. A player is the resolution creators in the game. In cognitive-femtocell networks, FBSs are treated as the players. The actions are the set of options accessible to every player. At any moment in time, a player should select a component from the set of actions. Usually, a set of actions may be distinct for dissimilar players. In cognitive-femtocell networks, the set of actions are generally the selection of modulation coding scheme (MCS), bit rate, network protocol, flow control metric, adaptive BS transmit power etc. Whilst any player selects an action, the leading "action profile" decides the consequences of the game. Generally, priorities are provided by defining a payoff function. A larger numerical value of payoff function signifies that the consequence is greater important in contrast to a consequence with smaller payoff function. The authoritative order, basically strategy of the BS within the HetNets is its transmission power or can be its area of network coverage. The payoff function may change depending upon two parameters, i.e., the range of the network coverage and the interference cause to the MUE. This correlates to the matter in which BSs are unable to carry out power control throughout the mechanism of the network. In this analytical framework, for

\footnotetext{
${ }^{1} \mathrm{~A}$ macro user equipment (MUE) is a device used directly by an end-user of macrocell to communicate.
} 
broader respect, we further consider that there are $N$ number of macro networks. Let, $K_{t o t}$ number of femtocells and $I_{t o t}$ FUEs directly served by MBS in cogntive femtocell networks. In general, the number of FBSs and FUE are appealing to get connected to MBS is large, which is, $\left(K_{t o t}+I_{t o t}\right) \geq N$ [19]. In every time slot, while a specific spectrum $W_{p}$ is utilised by an FBS, the goal of the FBS is to optimize its EE transmissions by assigning its power. Likewise, when the band is utilised by an MUE, an MBS does the energy-efficient power assignment. Depending upon the above exchange of views, the issue of resource allocation can be formulated for energy-efficient transmission in HetNets as a non-cooperative game problem, as explained in the following subsection.

Besides, few considerations that have been chosen as below: 1) In every macrocell OFDMA network, the number of channels is relatively greater size compared to the users' count on every occasion and no channel can concurrently facilitate transmission for more than one UE. Thus, entire UEs can concurrently transmit data to the FBS on a single channel or more than one channel in the absence of interference.

2) A UE is enjoy the network service by only one FBS that located within the same cell

3) The FBS cyclically approximates the downlink link gains on all channels for the entire UEs via pilot signals. Hence, the entire required channel state information can be correctly followed by the FBS.

The SINR of an FUE positioned in the 1st cell (i.e., FBS1 in Fig. 1), can be expressed as:

$$
\gamma_{1}=\frac{P_{1} h_{11} d_{11}^{-\alpha}}{P_{2} h_{12} d_{12}^{-\alpha}+N_{0}}
$$

where $P_{i}$ indicates the transmission power of $i^{\text {th }} \mathrm{FBS} ; h_{11}$ and $h_{12}$ represent small scale fading (SSF) between an FUE located at $1^{\text {st }}$ cell and its serving FBS, and between interfering FBS and an FUE located at $1^{\text {st }}$ cell respectively; $d_{11}^{-\alpha}$ and $d_{12}^{-\alpha}$ represent large scale path loss (LSPL) between an FUE located at $1^{\text {st }}$ cell and its serving FBS, and between interfering FBS and an FUE located at $1^{\text {st }}$ cell respectively; $\alpha$ stands for pathloss exponent. Likewise, SINR of an FUE positioned in the $2^{\text {nd }}$ cell (i.e., FBS2 in Fig. 1) is expressed as:

$$
\gamma_{2}=\frac{P_{2} h_{22} d_{22}^{-\alpha}}{P_{1} h_{21} d_{21}^{-\alpha}+N_{0}}
$$

where $h_{22}$ and $h_{21}$ represents SSF between an FUE located at $2^{\text {nd }}$ cell and its serving FBS, and between interfering FBS and an FUE located at $2^{\text {nd }}$ cell respectively; $d_{22}^{-\alpha}$ and $d_{21}^{-\alpha}$ represents LSPL between an FUE located at $2^{\text {nd }}$ cell and its serving FBS, and between interfering FBS and an FUE located at $2^{\text {nd }}$ cell respectively.

If an FUE is under the network coverage of its respective FBS, then quite obvious that the FUE will have a large enough SINR, i.e., $\gamma_{i} \geq \bar{\gamma}$ Therefore, at the edge area of the network coverage, we have

$$
\gamma_{i}=\bar{\gamma}
$$

The network coverage radius of $i^{t h}$ FBS is indicated by $r_{i}$. Let FUE1 and FUE2 are positioned at the border of the network coverage of FBS1 and FBS2 respectively. Then, we have

$$
\begin{aligned}
& \bar{\gamma}=\frac{P_{1} h_{11} r_{1}^{-\alpha}}{P_{2} h_{12}\left(D-r_{1}\right)^{-\alpha}+N_{0}}, \\
& \bar{\gamma}=\frac{P_{2} h_{22} r_{2}^{-\alpha}}{P_{1} h_{21}\left(D-r_{2}\right)^{-\alpha}+N_{0}},
\end{aligned}
$$

where, $D$ denotes location gap between FBS1 and FBS2. If an FUE is positioned at $r_{1}$ from the FBS1, its location gap from the FBS2 may be any integer value between $D-r_{1}$ and $D+r_{1}$. In (4), $D-r_{1}$ replacing $d_{12}$ in (1). Likewise, in (5), $D-r_{2}$ replacing $d_{21}$ in (1). Besides, we need that the total amount of interference produced by the two FBSs to the MBS should not be more than a pre-specified threshold $(\bar{\xi})$, i.e.,

$$
P_{1} h_{p 1} d_{p 1}^{-\alpha}+P_{2} h_{p 2} d_{p 2}^{-\alpha} \leq \bar{\xi}
$$

In order to address the existence of positive $P_{1}$ and $P_{2}$, the condition should be met as

$$
h_{11} r_{1}^{-\alpha} h_{22} r_{2}^{-\alpha}-\bar{\gamma}^{2} h_{12}\left(D-r_{1}\right)^{-\alpha} h_{21}\left(D-r_{2}\right)^{-\alpha} \neq 0,
$$

then, the solution for (4) and (5) derived as:

$$
\begin{aligned}
P_{1} & =\frac{\bar{\gamma} N_{0}\left[h_{22} r_{2}^{-\alpha}+\bar{\gamma} h_{12}\left(D-r_{1}\right)^{-\alpha}\right]}{h_{11} r_{1}^{-\alpha} h_{22} r_{2}^{-\alpha}-\bar{\gamma}^{2} h_{12}\left(D-r_{1}\right)^{-\alpha} h_{21}\left(D-r_{2}\right)^{-\alpha}}, \\
P_{2} & =\frac{\bar{\gamma} N_{0}\left[h_{11} r_{1}^{-\alpha}+\bar{\gamma} h_{21}\left(D-r_{2}\right)^{-\alpha}\right]}{h_{11} r_{1}^{-\alpha} h_{22} r_{2}^{-\alpha}-\bar{\gamma}^{2} h_{12}\left(D-r_{1}\right)^{-\alpha} h_{21}\left(D-r_{2}\right)^{-\alpha}} .
\end{aligned}
$$

In general, the power transmission of a BS and its network service depends upon the coverage radius of the other BSs. As distance $r_{1}$ and $r_{2}$ both are smaller than the distance $D$, hence numerator part of (8) and (9) are non-negative. Likewise, the denominator must be non-negative to have positive solutions $P_{1}$ and $P_{2}$, i.e.,

$$
h_{11} r_{1}^{-\alpha} h_{22} r_{2}^{-\alpha}-\bar{\gamma}^{2} h_{12}\left(D-r_{1}\right)^{-\alpha} h_{21}\left(D-r_{2}\right)^{-\alpha}>0,
$$

Or, equivalently:

$$
\left(\frac{h_{12}}{h_{11}}\right)\left(\frac{D-r_{1}}{r_{1}}\right)^{-\alpha}\left(\frac{h_{21}}{h_{22}}\right)\left(\frac{D-r_{2}}{r_{2}}\right)^{-\alpha}<\bar{\gamma}^{-2} .
$$

The above inequality (10) and (11) brings into use to obtain a realizable area of the network coverage region of two FBSs. If (11) holds, $P_{1}$ and $P_{2}$ can be obtained from (8) and (9). Additionally, $P_{1}$ and $P_{2}$ should satisfy the condition in (6). The payoff function for individual BS can be expressed as follows:

$$
u_{i}=\frac{r_{i}}{\left(\frac{D}{\sqrt{2}}\right)}-\frac{P_{i} h_{p i} d_{p i}^{-\alpha}}{\bar{\xi}},
$$

where $r_{i}$ is the radius of the femtocell corresponds to $F B S_{i}$, $P_{i}$ is the power transmission of $F B S_{i}$ and $d_{p i}$ is the distance 
between FBSs and the MBS. The payoff function is composed of only two parts. The positive part indicates the normalized network coverage of the FBS. The negative part indicates the normalized interference because of this player to the MUE.The negative part is chosen to permit the far away BS to perform at a large power and to get wider network coverage. The target of each FBS is to optimize its network coverage by including the payoff function. When replacing $P_{i}$ in (12) by $P_{1}$ and $P_{2}$ formulated in (8) and (9), we have

$$
\begin{array}{r}
u_{1}=\frac{r_{1}}{\left(\frac{D}{\sqrt{2}}\right)}-\begin{array}{c}
\frac{\bar{\gamma} N_{0} h_{p 1} d_{p 1}\left[h_{22} r_{2}^{-\alpha}+\bar{\gamma} h_{12}\left(D-r_{1}\right)^{-\alpha}\right]}{\bar{\xi} h_{11} r_{\frac{1}{-\alpha} h_{22} r_{2}^{-\alpha}}}, \\
-\bar{\xi} \bar{\gamma}^{2} h_{12}\left(D-r_{1}\right)^{-\alpha} h_{21}\left(D-r_{2}\right)^{-\alpha}
\end{array} \\
\begin{array}{r}
u_{2}=\frac{r_{2}}{\left(\frac{D}{\sqrt{2}}\right)}-\frac{\bar{\gamma} N_{0} h_{p 2} d_{p 2}\left[h_{11} r_{1}^{-\alpha}+\bar{\gamma} h_{21}\left(D-r_{2}\right)^{-\alpha}\right]}{\bar{\xi} h_{11} r_{1}^{-\alpha} h_{22} r_{2}^{-\alpha}} \\
-\bar{\xi} \bar{\gamma}^{2} h_{12}\left(D-r_{1}\right)^{-\alpha} h_{21}\left(D-r_{2}\right)^{-\alpha}
\end{array}
\end{array}
$$

In order to get an accurate understanding into the strategic behavior of the players, we employ the best response concept. If mutual best responses are obtained by the two strategies, then no player would not have any inducement to differ from the assigned strategy profile. One can get the best response of individual player from the payoff functions introduced in (13) and (14).

Lemma: In the realizable part of an area, the payoff function corresponds to player $i$ is a concave function of $r_{i}$.

Proof : The concavity of $u_{i}$ is negative all the time in its feasible region can be shown by taking its $2^{\text {nd }}$ derivative. Now, $u_{2}$ can be expressed as:

$$
u_{2}=\frac{r_{2}}{\left(\frac{D}{\sqrt{2}}\right)}-\bar{\gamma} N_{0} d_{p 2}^{-\alpha} \bar{\xi}^{-1} \cdot \frac{A}{B},
$$

where,

$$
\begin{aligned}
& A=\left(h_{11} r_{1}^{-\alpha}+\bar{\gamma} h_{21}\left(D-r_{2}\right)^{-\alpha}\right), \\
& B=h_{11} r_{1}^{-\alpha} h_{22} r_{2}^{-\alpha}-\bar{\gamma}^{2} h_{12}\left(D-r_{1}\right)^{-\alpha} h_{21}\left(D-r_{2}\right)^{-\alpha}
\end{aligned}
$$

The $1^{\text {st }}$ derivative of $u_{2}$ is as follows:

$$
\frac{d u_{2}}{d r_{2}}=\frac{1}{D \sqrt{2}}-\bar{\gamma} N_{0} d p_{2}^{-\alpha} \bar{\xi}^{-1} \frac{\frac{d A}{d r_{2}} B-\frac{d B}{d r_{2}} A}{B^{2}} .
$$

The $2^{\text {nd }}$ derivative is:

$$
\begin{gathered}
\left(\frac{d^{2} A}{d^{2} r_{2}} B-\frac{d^{2} B}{d^{2} r_{2}} A\right) \\
\frac{d^{2} u_{2}}{d r_{2}^{2}}=-\bar{\gamma} N_{0} d p_{2}^{-\alpha} \bar{\xi}^{-1} \frac{-2 \frac{d B}{d r_{2}} B\left(\frac{d A}{d r_{2}} B-\frac{d B}{d r_{2}} A\right)}{B^{4}} .
\end{gathered}
$$

The $1^{\text {st }}$ derivative of $\mathrm{A}$ is:

$$
\frac{d A}{d r_{2}}=\bar{\gamma} h_{21} \alpha\left(D-r_{2}\right)^{-\alpha-1}
$$

By taking the $2^{\text {nd }}$ derivative on (19), we obtain:

$$
\frac{d^{2} A}{d r_{2}^{2}}=\bar{\gamma} h_{21} \alpha(\alpha+1)\left(D-r_{2}\right)^{-\alpha-2}
$$

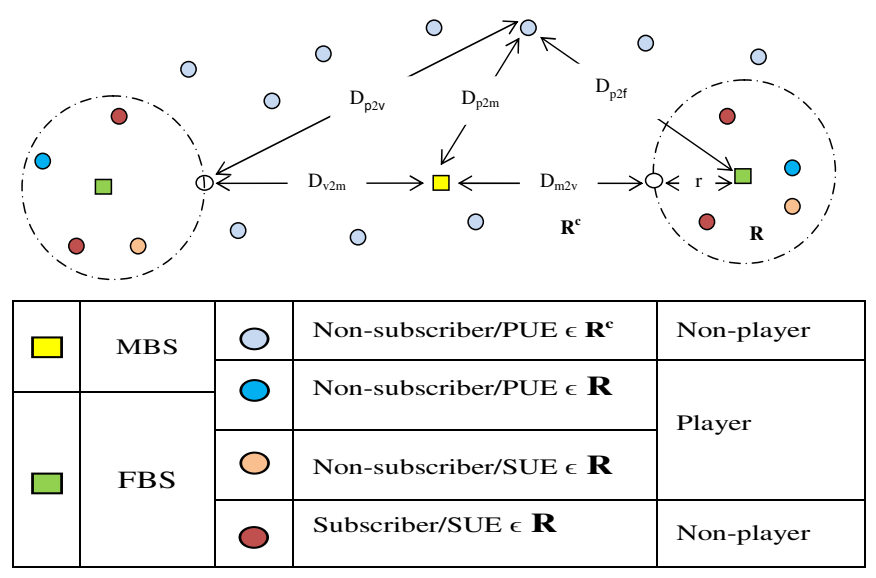

Fig. 2: The network exploration with role of different types of UEs in the game.

The $1^{\text {st }}$ derivative of $B$ is:

$$
\begin{aligned}
& \frac{d B}{d r_{2}}=-\alpha h_{11} r_{1}^{-\alpha} h_{22} r_{2}^{-\alpha-1} \\
& \quad-\alpha \bar{\gamma}^{2} h_{12} h_{21}\left(D-r_{1}\right)^{-\alpha}\left(D-r_{2}\right)^{-\alpha-1} .
\end{aligned}
$$

Similarly,

$$
\begin{aligned}
\frac{d^{2} B}{d r_{2}^{2}}=\alpha & (\alpha+1) h_{11} r_{1}^{-\alpha} h_{22} r_{2}^{-\alpha-2} \\
& \quad-\alpha(\alpha+1) \bar{\gamma}^{2}\left(D-r_{1}\right)^{-\alpha}\left(D-r_{2}\right)^{-\alpha-2} .
\end{aligned}
$$

From (16), as $r_{1}$ and $r_{2}$ are smaller than $\mathrm{D}$ and applying the condition of (10), we get: $A>0$ and $B>0,(a)$; from (19) and (20), we get: $\frac{d A}{d r_{2}}>0$ and $\frac{d^{2} A}{d r_{2}^{2}}>0,(b)$; $\frac{d B}{d r_{2}}<0$ and $\frac{d^{2} B}{d r_{2}^{2}}>0,(c)$; from (16), as $A-B>0$, we get: $A>B,(d)$; from (19) and (21), as $\frac{d B}{d r_{2}}-\frac{d A}{d r_{2}}>0$, we get: $\frac{d B}{d r_{2}}>\frac{d A}{d r_{2}}$, (e); from (22) and (23), as $\frac{d^{2} A}{d r_{2}^{2}}-\frac{d^{2} B}{d r_{2}^{2}}>0$, we get: $\frac{d^{2} A}{d r_{2}^{2}}>\frac{d^{2} B}{d r_{2}^{2}},(f)$; from (18), applying the conditions ((a) to (f)), we get: $\frac{d^{2} u_{2}}{d r_{2}^{2}}<0, \frac{d^{2} u_{1}}{d r_{1}^{2}}<0,(g)$. The obtained conditions in (g) signify that $u_{i}$ is a concave function of $r_{i}$.

Fig. 2 describes cognitive femtocell networks of different access strategies and the role of activity of different types of users. Suppose $R$ and $R_{C}$, respectively, represent sets of users that locate inner places and outer place of the upper transmission limit of the FBS. The subscribers assumed in $R$ having the authority to access their subscribing FBS. The non-subscribers are categorized depending upon their positions either in $R$ or $R^{C}$. As non-subscribers in $R^{C}$ are capable to choose the macrocell as their target network entity, they are assumed independent of the game theory selection process. Moreover, non-subscribers in $R$ have the option to take the service from either network entity to obtain optimum payoffs. Although, subscribers are having the license to influence the game in spite of the fact that they are not players in the game. Besides, based on cognitive radio theory, both the nonsubscribers $\in R^{C}$ and the players which access the MBS are 
treated as PUs; whereas accessing the FBS network is treated as SUs.

Remark 1. $\mathrm{U}$ to be the total users and $U \in R$ are positioned at the edge of the FBS network coverage.

Remark 2. The non-subscribers that belong to the same femtocell have equal scheduling importance, and next perform for the other non-subscribers that are belonging to the macrocell.

All users $\in R$ mentioned in Remark 1 are substituted by a virtual user (say node $V$ ) as shown in Fig. 2. The position of node $V$ correlates to the shortest path from MBS to any position within network coverage of FBS. $D_{v 2 m}$ or $D_{m 2 v}$ stands for distance between an FUE positioned at node $V$ and the MBS, $D_{p 2 v}$ indicates distance between virtual node $V$ and an MUE, $D_{p 2 f}$ represents distance of an MUE from the FBS, $D_{p 2 m}$ is the notation to define distance between an MUE and the MBS. The case where node $V$ is linked to the FBS, user suffers from extreme interference and reduces the SINR of macrocell users in the downlink directions compare to other users $\in R$ which also connect to the femtocell. So that, the virtual UE $\mathrm{V}$ can be used to indicate all UEs $\in R$ in a more conventional way by including certain situation based restrictions. Besides, as per Remark 2, the scheduling method and priority of the non-subscribers are considered to be same within the service network irrespective of MBS or FBS. As in [20], the following game is reformed to formulate the issue of spectrum allocation among the PU and multiple SUs for the two-tier network. Let us consider $\mathrm{H}$ subscribers $\in R$ and $H^{c}$ non-subscribers $\in R^{c}$ in this analytical framework. Furthermore, we assume $\epsilon$ as an admission control parameter in this paper. It indicates that all subscribers can initially be assigned with the ratio $\epsilon$ of the entire capacity $C_{f}$ for the FBS, and all the FUs will use rest $(1-\epsilon)$ of the capacity. Suppose $\mu_{f}(n)$ be explicated as the utility of individual subscriber, which is possible to achieve by

$$
\mu_{f}(n)=\left(\frac{\epsilon}{\mathbf{H}}+\frac{1-\epsilon}{\mathbf{H}+\mathbf{n}}\right) C_{f} .
$$

If $\epsilon=1, \mu_{f}(n)=\frac{\epsilon}{\mathbf{H}} C_{f}$ indicates that the femtocell is totally closed. Similarly, if $\epsilon=0, \mu_{f}(n)=\frac{1}{\mathbf{H}+\mathbf{n}} C_{f}$ indicates that the femtocell is totally opened.

Large transmission power (TP) at an FBS can very badly lower the efficiency of other hugely-loaded FBSs and MBSs due to extreme intra-tier and inter-tier interference. To cope up with this problem, we introduce an effective power adjustment algorithm by utilizing game theory. An objective of power adjustment algorithm is to adjust TP of FBS to network's traffic load [8]. Hence, this can only be enabled whilst the traffic load gives a detailed account of user arrival rate changes. Every FBS $i$ can choose its entire TP, $P_{i} \in\left[0, P_{F B S}^{\max }\right]$, under the strategies to make the best use of its payoff. Here, the transmission power ratio is denoted by $\beta_{i}$ for FBS $i$ is in $[0,1]$. Payoff of $F_{i}\left(P_{i}, P_{-i}\right)$ for every FBS $i$ is $F_{i}\left(P_{i}, P_{-i}\right)=-P_{i}$, where $P_{-i} \triangleq\left[P_{1}, \cdots, P_{i-1}, \cdots, P_{i+1}, \cdots, P_{J}\right]$ indicates a vector holding TPs of other FBSs excepting FBS $i$. In the end, we consider that FUE has the highest tolerable blocking probability (BP), $P_{b}^{f h}$. Let $P_{i c}^{f}$ indicates the BP of a FUE located in the network coverage of femtocell $i$ in closed access mode. Thus, the BP condition can be expressed as:

$$
P_{i c}^{f} \leq P_{b}^{f h}, \quad \forall i .
$$

The admission control and channel allotment to be operated according to the requirement to fulfill the BP condition, whilst optimizing their payoffs. Besides, we need that every Femtocell that wants to reduce its TP should obey the following BP condition for their macro user equipments (MUEs) as:

$$
P_{i c}^{m} \leq P_{b}^{m h}, \quad \forall i,
$$

provided that these conditions are fulfilled when the highest power, $P_{F B S}^{\max }$ is employed. Here, $P_{b}^{m h}$ indicates some preset value. Particularly, we permit moderately-loaded femtocells to decrease their TPs, result in enhancement of the BPs to its FUs until the BPs fulfill the conditions in (24) and (25). The decrease in TPs for lightly-loaded femtocells will enhance transmission rates (TRs) of highly-loaded FBSs. This is substantially decreasing the channel necessities, which in turn improve BPs, SE and EE in these highly-loaded FBSs. The admission control performance (ACP) by means of channel necessity can be adjusted by the following principle of Femtocell Power Adaptation Algorithm (FPAA).

Definition: The state of equilibrium of the non-cooperative is a power profile, i.e., $P^{*} \in\left[0, P_{F B S}^{\max }\right]$, of the strategies, such that:

$$
F_{i}\left(P_{i}^{*}, P_{-i}^{*}\right) \geq F_{i}\left(P_{i}, P_{-i}^{*}\right),
$$

where $\geq$ stands for priority correspondence on payoffs of strategies such as $P_{i}^{*}$ and $P_{i}$. The equilibrium can be instinctively explained as if $F_{i}$ gives a strategy proposal from the reliable third-party to $i$ player. Then, the implied consideration is that the rest of the players obey this proposal, and $i$ players implore themselves for their finest regard to obey the proposal. The equilibrium state signifies that there is no player having any strategy which can produce a greater payoff compared to one for playing $P_{i}^{*}$, provided that each rest player played their strategy for $P_{-i}^{*}$.

Convergence Analysis: The notation $x^{a} \in \triangle \mathbb{A}$ is the distribution function associated with the empirical measure of an ordered set of strategies run up to time $a$. Its component, symbolized by $x^{a}(A), \forall A \in \mathbb{A}$ indicates the relative frequency where $A$ has been played for the time $a$,i.e.,

$$
x^{a}(A)=\frac{1}{a}\left|t \leq a: A^{t}=A\right| .
$$

Besides, the distribution function associated with the empirical measure, symbolized by $x^{a}$, can achieve by the a recursive procedure [27]:

$$
x^{a+1}=x^{a}+\frac{1}{a+1}\left(e_{A^{a+1}}-x^{a}\right),
$$

where $e_{A^{a+1}}=[0,0, \cdots, 1,0 \cdots, 0]$ indicates the $|\mathbb{A}|$ dimensional unit vector with 1 in the place of $A^{a+1}$.

Remark 3: If each player obeys the introduced algorithm, the convergence is going to be achieved for $a \rightarrow \infty$ at the state of equilibrium [27]. 


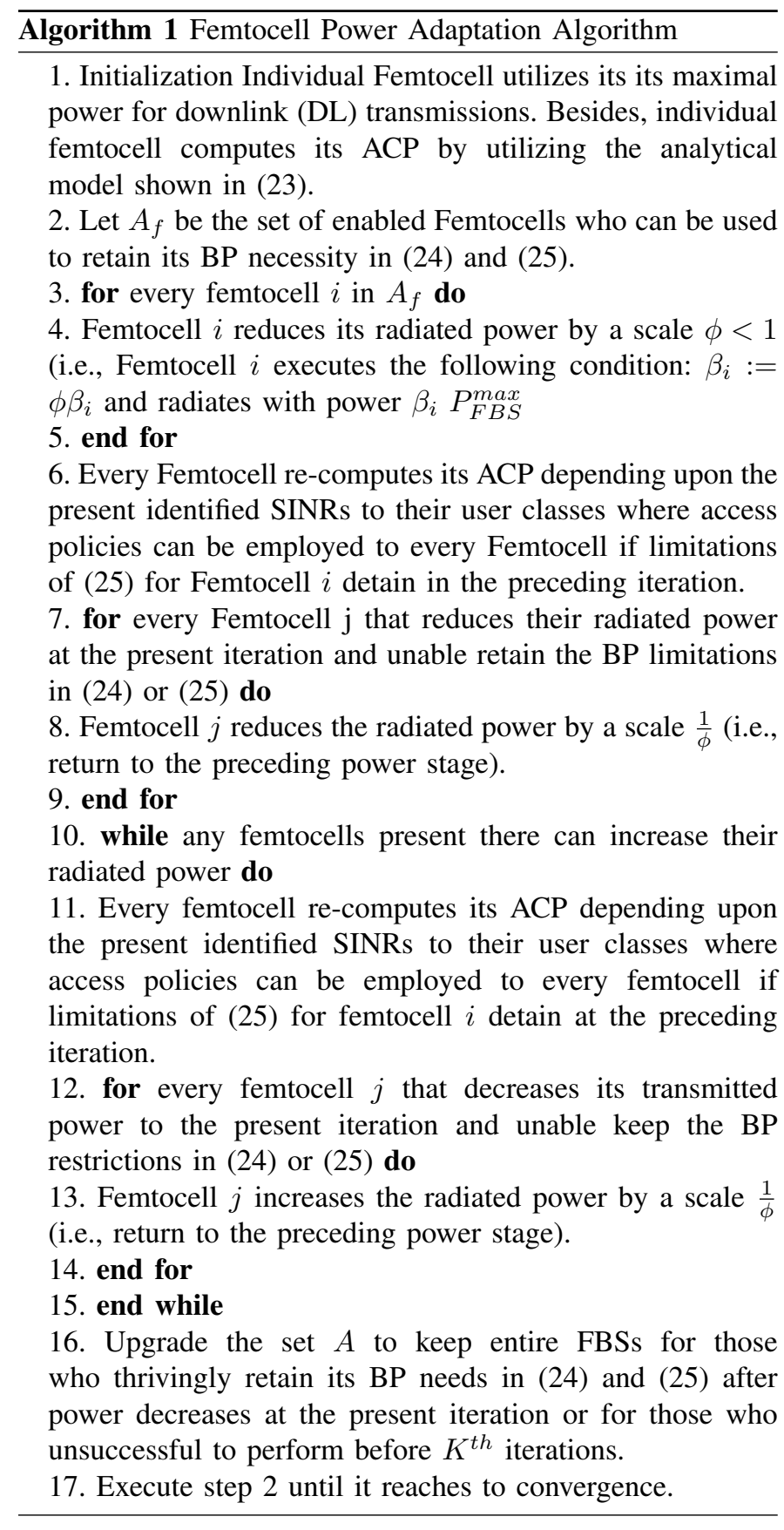

Complexity Analysis: An individual player requires to retain a record of the payoff of selecting the present strategy and the payoffs for altering to different strategies at every iteration. Additionally, the algorithm necessitates a lookup table for not less than $\left(a+n_{f} n_{u}\right)$ times insertions and $\left(n_{f} n_{u}+1\right)$ times product to avoid the erroneous values and a differentiation to select the succeeding strategy. Here, $n_{f}$ denotes number of FBSs and $n_{u}$ denotes number of UEs. The complexity order dependent on the strategy counts, i.e., $O\left(\Omega_{n_{f}}\right)$ where $\Omega$ indicates the strategy space for $n_{f}$ number of players, which is obtained by following the similar type of analytical work given in [28].

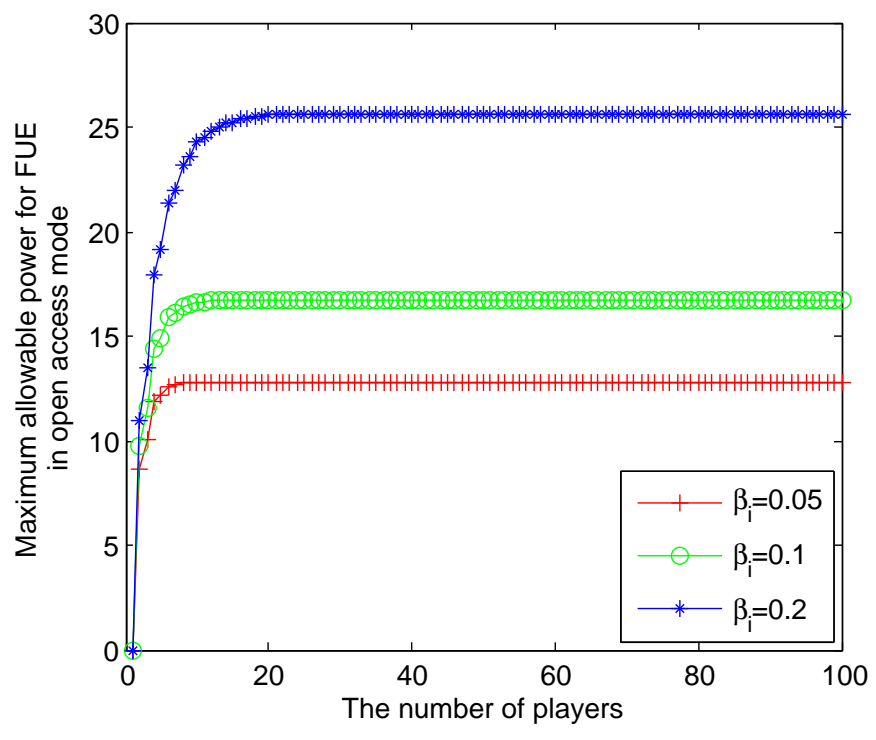

$3(\mathrm{a})$

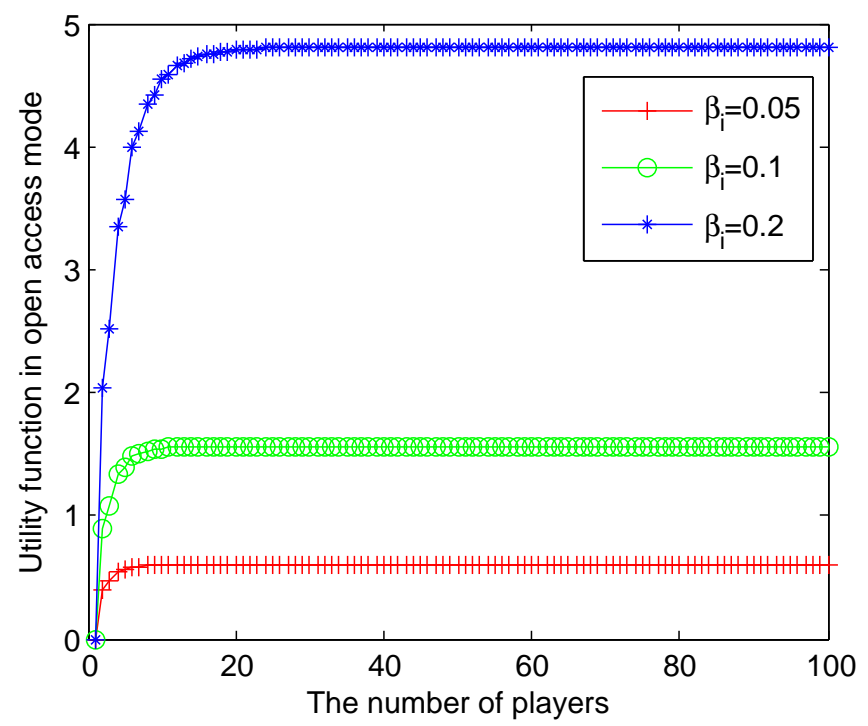

3(b)

Fig. 3: (a) Influence of adaptive transmission power in open access mode, (b) Influence of utility optimization in open access mode.

\section{Numerical Results And Discussion}

The numerical results have been chosen to demonstrate the concavity response of price weighting factor $\left(\beta_{i}\right)$ and the convergence of algorithm. In the simulations, spectrum allocation and MUs are randomly distributed in the range of MBS, and cognitive FUs are uniformly distributed in the network coverage of their serving femtocell; carrier frequency $2.5 \mathrm{GHz}$, $\mathrm{BW}=20 \mathrm{MHz}$, AWGN power spectral density $N_{0}=-174$ $\mathrm{dBm} / \mathrm{Hz}$.

The evolution of adaptive transmission power, utility optimization, and interference with the increase number of players in open access mode and closed access mode over the upgradation of the synchronous power have been performed and it depicts the convergence of algorithm in Figs. 3, 4, and 5, 


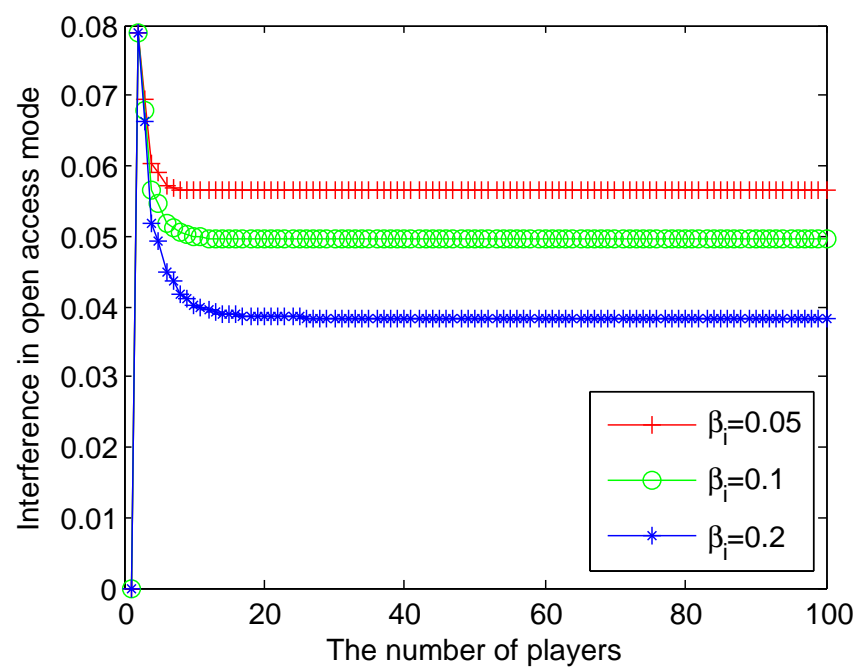

4(a)

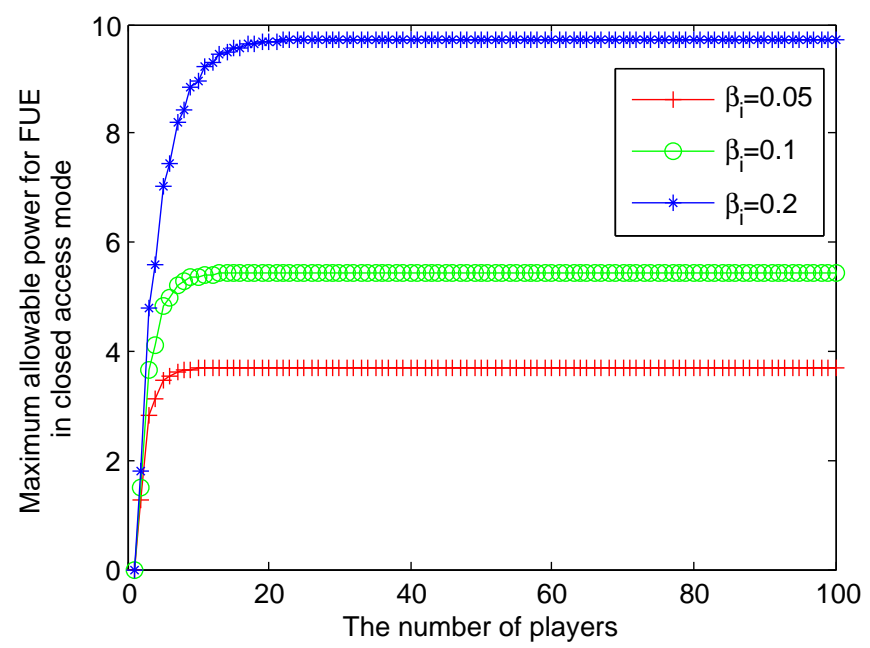

4(b)

Fig. 4: (a) Influence of interference with the increase in no of players in open access mode, (b) Influence of adaptive transmission power in closed access mode

respectively. In Figs. 3, 4, and 5, the convergence of algorithm is computed with the blocking probability constraint $=0.01$, the inter-tier interference limit $=6.5 \times 10^{-14} \mathrm{~W}$, minimum data rate needs $=0.5 \mathrm{bps} / \mathrm{Hz}$. As it can be shown from Figs. 3, 4, and 5, maximum allowable power for FUE in sub-channel, utility function and overall all interference in open access mode and closed access mode converge after reaching the no. of players equals to 15, respectively. Fig.3(a), 3(b) and 4(a) show maximum allowable power, utility function, and interference versus the number of players that are connected to the FBS in open access mode. It is interesting to perceive that the utility function for the subscribers get larger with the greater number of players that are connected to the femtocell under $\beta_{i}=0.05,0.1,0.2$. The total capacity of subscribers is grown by allocating specific amount of spectrum capacity with the non-subscribers because those non-subscribers decide

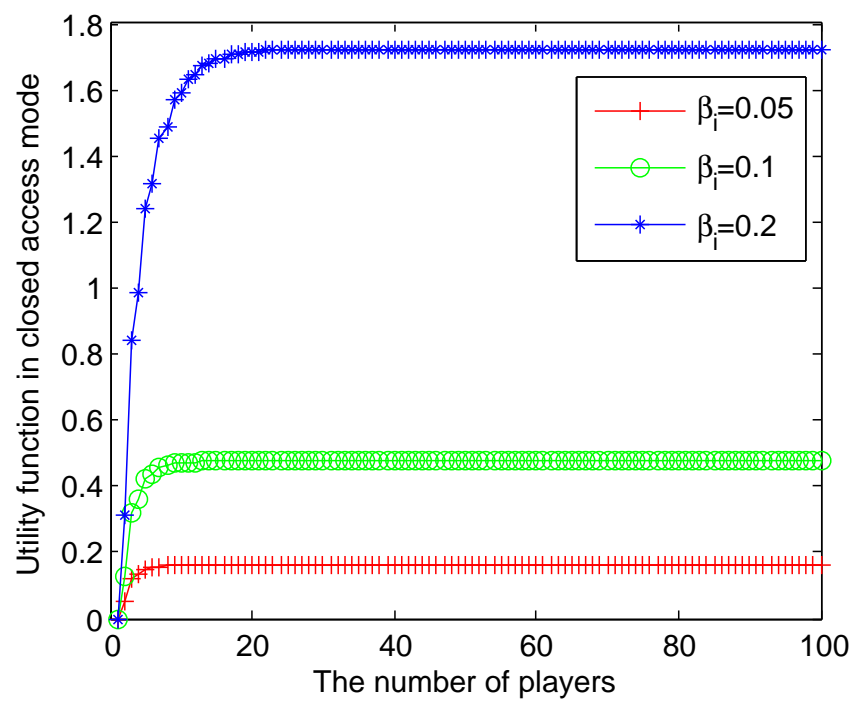

$5(a)$

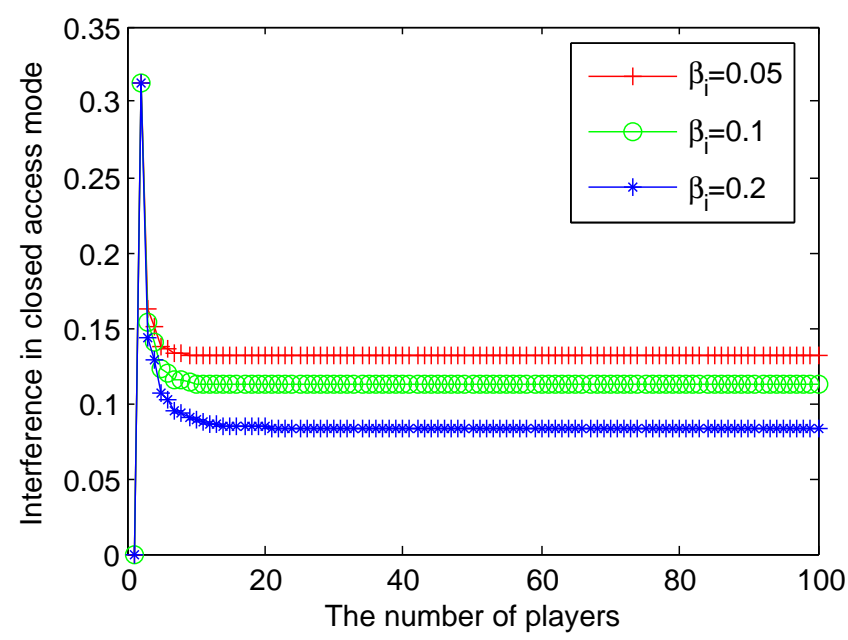

5 (b)

Fig. 5: (a) Influence of utility optimization in closed access mode, (b) Influence of interference with the increase in no of players in closed access mode

not to connect to the macrocell. Fig. 4(a) illustrates the interference function achieved from (6) versus number of players under $\beta_{i}=0.05,0.1,0.2$ and it can also be found that the interference is less sensitive to the change of $\beta_{i}$, but the interference reduces dramatically when number of players higher than 2. If the power consumption price smaller than a certain limiting level, then a FBS decides to transmit as the payoff higher than the price. Hence, a very low power consumption price weighting factor can have a positive effect on the interference response. Considering closed access mode where $\epsilon=1$, it can be noticed that the maximum allowable power of subscribers grows for $n=1$ to around 10 , and reduces with the decrease in fixed value corresponding to $\beta_{i}$. Within the transient portion of utility function from $n=1$ to 10 , the subscribers permit all the players to use the network of 


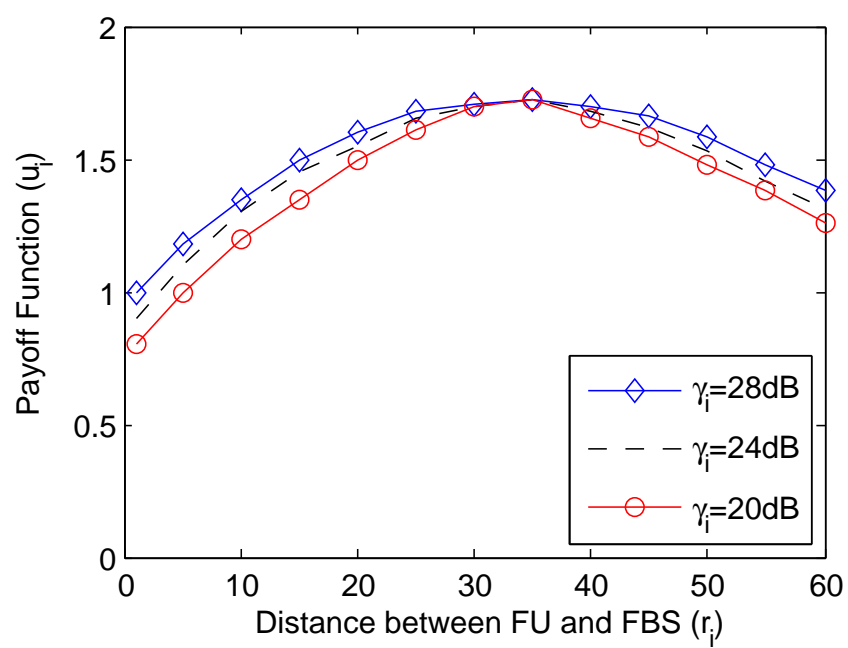

6(a)

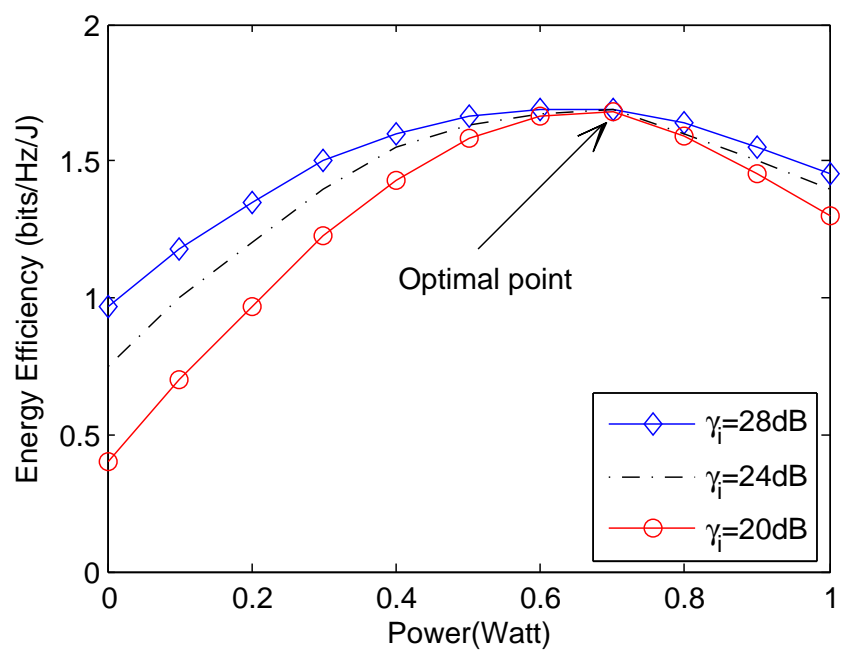

6(b)

Fig. 6: (a) Concavity response of payoff function $\left(u_{i}\right)$ in accordance with change in coverage range $\left(r_{i}\right)$, (b) Concavity response of energy efficiency under optimal power transmission keeping $\phi$ fixed at 2 .

FBS as exhibited in Fig. 5(a). Fig. 5(b) illustrates that the interference in closed access mode is highest when there are number of players equal to 2 , and thereafter drastic fall in interference can be seen with the increase in number of players. From the Fig. 6(a), this can observe that as SINR of a FUE within the femtocell increases, the payoff function of the dual layer cognitive femtocell network increases. This is due to the fact that the FBS gets shorter transmission range, that has more possibility to receive large transmission rate with small power because of good channel condition. Additionally, it is known that as the gap between FUE and FBS increases, the channel condition becomes poorer quality, and more amount of power is required. Thus the payoff function of the network, when distance between FUE and FBS cross certain limiting distance, responds concave downward. The act of carrying out performance evaluation of FBS by considering its energy efficiency on top of the power transmission is presented in Fig. 6(b) and 7. From these figures, this finds that the energy efficiency initially grows with increase in its power, and while the power reaches optimal point, the energy efficiency starts to reduce. Due to this, there is a balanced achieved for the energy-efficient power assignment between two desirable but incompatible features such as transmission capacity and power consumption. This finds from the results that small amount of transmitting power is required for the femtocell with better channel state information to achieve equal energy efficiency for the femtocell, which means, more the channel state information, better the energy efficiency possible to achieve in the femtocell. Figures 6 and 7 demonstrate that regardless of how many UEs are linked to the DL transmission and FBSs are deployed, the equilibria is possible to reach through the algorithm. This can be perceived that at least 150 iteration is the minimum necessary condition to reach the convergence. Besides, the large deployment of FBSs or the large accumulation of UEs within the network coverage, leads to sluggish response in the convergence. Furthermore, it is to be observed that the rate of convergence alters with the radiated power factor, distance between an FU and a FBS, and SINR.

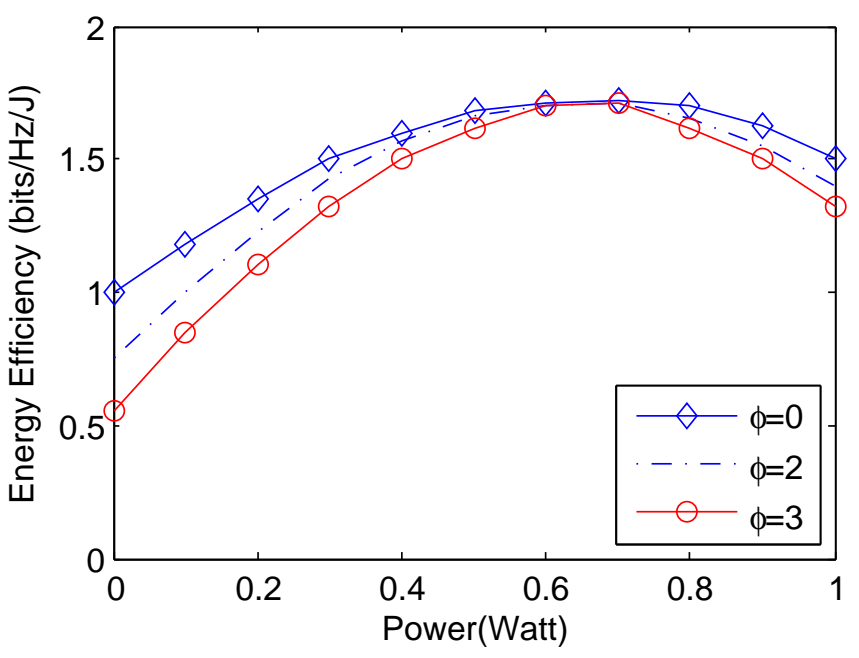

Fig. 7: Concavity response of energy efficiency under optimal power transmission keeping $\gamma_{i}$ fixed at $25 \mathrm{~dB}$.

\section{CONCLUSION}

In this paper, an analytical non-cooperative game theoretic framework has been developed by following the proper FBSs' resource allocation where an FBS performs as a game player to find the most satisfying subscriber and studied the difficulties of power assignment in heterogeneous networks. By applying a non-cooperative game theoretic method, the strategic components for the FBSs have detected in developed network model. Besides, an efficient femtocell power algorithm has been introduced in cognitive femtocell scenario, in which EE and payoff function converge to optimal point at the equilibrium. In order to reduce the interference as well as to improve the $\mathrm{EE}$ in the future generation networks, concave downward reasoning of the payoff function has been proven 
for balancing the EE that could not fail to be stimulated by the idea presented.

\section{REFERENCES}

[1] F. R. Yu, X. Zhang, and V. C. M. Leung, Green Communications and Networking. New York: CRC Press, 2012.

[2] Y. Chen, S. Zhang, S. Xu, and G. Y. Li, "Fundamental trade-offs on green wireless networks," IEEE Comm. Mag., vol. 49, no. 6, pp. 3037, Jun. 2011

[3] G. Gur and F. Alagoz,"Green wireless communications via cognitive dimension: an overview," IEEE Network, vol. 25, pp. 5056, Mar. 2011.

[4] Hui Li, Dario Landa-silva, Xavier Gandibleux, "Evolutionary multiobjective optimization algorithms with probabilistic representation based on pheromone trails," Evolutionary Computation (CEC) 2010 IEEE Congress on, pp. 1-8, 2010.

[5] P. Si, H. Ji, F. R. Yu, and V. C. M. Leung, "Optimal cooperative internetwork spectrum sharing for cognitive radio systems with spectrum pooling,” IEEE Trans. Veh. Tech., vol. 59, no. 4, May 2010.

[6] F. R. Yu, B. Sun, V. Krishnamurthy, and S. Ali, "Application layer QoS optimization for multimedia transmission over cognitive radio networks," Wirel. Netw., vol. 17, pp. 371383, Feb. 2011.

[7] Joydev Ghosh, Dushantha Nalin K. Jayakody,“An Analytical View of ASE for Multi-cell OFDMA Networks Based on Frequency Reuse Scheme," IEEE Systems Journal, June 2018.

[8] Nasir Faruk, Kalle Ruttik, Edward Mutafungwa, Riku Jntti, "Energy savings through self-backhauling for future heterogeneous networks," Energy, Volume 115, Part 1, 15 November 2016, Pages 711-721.

[9] Zoltan Jako, Joydev Ghosh, "Network Throughput and Outage Analysis in a Poisson and Matrn Cluster based LTE-Advanced Small Cell Networks," International Journal of Electronics and Communications, Vol.75, May 2017, Pages 4652.

[10] Y. Liang, W. Chung, G. Ni, I. Chen, H. Zhang, S. Kuo,"Resource allocation with interference avoidance in OFDMA femtocell networks," IEEE Transactions on Vehicular Technology 2012; 61(5):22432255

[11] W. Cheung ,T. Quek, and M. Kountouris, "Throughput optimization, spectrum allocation, and access control in two-tier femtocell networks," IEEE Journal on Selected Areas in Communications2012; 30(3):561574.

[12] J. Mitola,"Cognitive radio: an integrated agent architecture for software defined radio," Ph.D Thesis, KTH Royal Institute of Technology, Stockholm, Sweden, 2000

[13] J. He, C. Xu, L. Li,"Adaptive subcarrier bandwidth and power in OFDMbased cognitive radio systems for high mobility applications," International Journal of Communication Systems 2012; DOI: 10.1002/dac2424.
[14] N. Saquib, E. Hossain, L. Le, D. Kim, "Interference management in OFDMA femtocell networks: issues and approaches," IEEE Wireless Communications 2012; 19(3):8695.

[15] G. Gr, F. Alagz,"Green wireless communications via cognitive dimension: an overview," IEEE Network 2011; 25(4):5056.

[16] S. Maharjan, Y. Zhang, and S. Gjessing, "Economic Approaches for Cognitive Radio Networks: A Survey," Wireless Pers. Commun., vol. 57, no. 1, pp. 3351, Mar. 2011.

[17] Chunyan An, Renchao Xie, Hong Jiand Yi Li, "Pricing and power control for energy-efficient radio resource management in cognitive femtocell networks" Journal of Communication Systems Volume 28 , Issue 4, pages 743761, 10 March 2015.

[18] M D Weng, B H Lee, and J M Chen,"Two novel price-based algorithms for spectrum sharing in cognitive radio networks," Eurasip Journal Wireless Communications and Networking.

[19] S. Buzzi, G. Colavolpe, D. Saturnino, A. Zappone, "Potential games for energy-efficient power control and subcarrier allocation in uplink multicell OFDMA systems," IEEE J. Topics Signal Process., vol. 6, no. 2, pp. 89-103, Apr. 2012.

[20] Madhu Sudan Dahal, Jagan Nath Shrestha, Shree Raj Shakya, "Energy saving technique and measurement in green wireless communication," Energy, Volume 159, 15 September 2018, Pages 21-31.

[21] Ahmad AlAmmouri, Jeffrey G. Andrews, Franois Baccelli, "SINR and Throughput of Dense Cellular Networks With Stretched Exponential Path Loss," IEEE Transactions on Wireless Communications, vol. 17, no. 2, pp. 1147-1160, 2018.

[22] Joydev Ghosh, Dushantha Nalin K. Jayakody, "Game Theoretic Frequency Reuse Approach in OFDMA Femtocell Networks," Trans. on ETT 2018.

[23] J. Moura, and D. Hutchison,"Game Theory for Multi-Access Edge Computing: Survey, Use Cases, and Future Trends", IEEE Communications Surveys Tutorials, Vol. 21 , No. 1, pp. 260 - 288, 2019.

[24] C. Jiang, H. Zhang, Y. Ren, Z. Han, K-C Chen, L. Hanzo, "Machine Learning Paradigms for Next-Generation Wireless Networks", IEEE Wireless Communications, vol. 24, no. 2, pp. 98-105, 2017.

[25] D. T. Hoang, X. Lu, D. Niyato, P. Wang, D. I. Kim, and Zhu Han, "Applications of Repeated Games in Wireless Networks: A Survey", IEEE Communications Surveys Tutorials, Vol. 17 , No. 4, pp. 2102 2135,2015

[26] C. Jiang, H. Zhang, Y. Ren, H-H Chen,"Energy-Efficient Noncooperative Cognitive Radio Networks: Micro, Meso and Macro Views", IEEE Communications Magazine, vol. 52, no. 7, pp. 14-20, 2014.

[27] S. Hart, A Mas-Colell, "A simple adaptive procedure leading to correlated equilibrium" Econometrica, vol.68, no. 5, pp.11271150, 2000.

[28] B Wang, Z Han, KJR Liu, " Peer-to-peer file sharing game using correlated equilibrium” IEEE ACISS, pp. 729734, June 2009. 